\title{
Surface Modification of Stainless Steel by Coating with DLC/Ti Bi-Layered Film Using Sputter-Deposition
}

\author{
Tsutomu Sonoda, Setsuo Nakao and Masami Ikeyama \\ National Institute of Advanced Industrial Science and Technology (AIST) \\ 2266-98 Anagahora, Shimoshidami, Moriyama-ku, Nagoya \\ Fax: 81-52-736-7400, e-mail: tsutomu.sonoda@aist.go.jp
}

\begin{abstract}
Surface modification of stainless steel was examined by coating with DLC/Ti bi-layered films using sputter-deposition, in order to improve not only the abrasion resistance of the stainless steel but also the adhesion between the deposited DLC film and the steel surface by relaxing the stress concentrated at the interface between them. For the comparison, the sputter-deposition of DLC monolithic films onto the stainless steel was also performed under the same sputtering conditions as for depositing the DLC layers of the bi-layered films. Under visual observation, the obtained DLC/Ti bi-layered films appeared to be adhesive while the DLC monolithic films deposited directly onto the substrates peeled off partly. Thus it was found that the DLC/Ti bi-layered films were more adhesive to the stainless steel than the DLC monolithic films. According to Raman spectroscopy, it was found that a typical spectrum for DLC coatings was detected for the obtained DLC/Ti bi-layered film, confirming that DLC coatings were also formed on the titanium layer. Furthermore, according to indentation hardness measurements, the surface hardness of the DLC/Ti bi-layered films was much higher than that of the stainless steel substrates while the surface hardness of the titanium films deposited on the stainless steel substrates was slightly lower than that of the stainless steel substrates. Thus it was found that the surface hardness of the stainless steel could be improved by coating it with the DLC/Ti bi-layered films. On the other hand, according to potentiodynamic polarization experiments, it was also found that the corrosion protective property of the stainless steel could be improved by this coating process. Therefore it was expected that both the abrasion resistance of the stainless steel and also the adhesion between the deposited DLC film and the steel substrate as well as the corrosion property could be improved at the same time.
\end{abstract}

Key words: diamond-like carbon, DLC coating, stainless steel, titanium, intermediate layer, sputtering, adhesion

\section{INTRODUCTION}

Diamond-like carbon (DLC) coatings feature excellent mechanical properties and chemical ones, such as high hardness, low friction coefficient, wear resistance and corrosion resistance, chemical stability, biocompatibility [1-3], etc. Thanks to these properties, the coatings are attractive for industrial applications. However, stress concentrations are caused at the interface between DLC coatings and metallic substrates. This brings about problems in adhesion and endurance life of the coatings subjected to mechanical loadings, e.g. tribological or thermal stress. In addition, hard DLC coatings have high compressive stress, and such high compressive stresses often lead to poor adhesion with substrates, especially with those of steel, which limits its use in practical applications. On the other hand, it is known that a thin intermediate layer such as titanium layer improves the adhesion between the coating and the substrate [4-6].

In the present work, therefore, the surface modification of stainless steel substrates with DLC/Ti bi-layered films by magnetron DC sputtering was examined, in order to improve not only both the abrasion resistance and the corrosion resistance at the same time but also the adhesion between the deposited film and the substrate by relaxing the stress concentrated at the interface between them.

\section{EXPERIMENTAL}

A planar magnetron sputtering system (ANELVA Corp. type L-332S-FHS) with 3 cathodes was used. The planar targets used for sputter-deposition of the bi-layered films were a pure titanium disk and a pure carbon disk of 80 $\mathrm{mm}$ in diameter. Stainless steel (SUS304) substrates were mounted on the water-cooled substrate holder. The stainless steel substrates were mirror-polished. The surface of the stainless steel substrates was cleaned by ultrasonic cleaning with organic solvent prior to coating. The substrates were grounded during the sputter-deposition The deposition of DLC/Ti bi-layered films onto the substrates (hereafter named DLC/Ti/SUS) was carried out in the atmosphere of argon at the pressure of $0.4 \mathrm{~Pa}$ by sputtering of a titanium target followed by 
sputtering a carbon one. DLC layers and titanium layers were respectively deposited under the condition that the substrates were located right above the carbon target and the pure titanium target. DC powers supplied for the sputtering of the titanium target and the carbon one were respectively around $50 \mathrm{~W}$ and $300 \mathrm{~W}$. The deposition times for the titanium film and the carbon one were respectively $5 \mathrm{~min}$ and $22 \mathrm{~min}$. For the comparison, the sputter-depositions of titanium monolithic films onto the stainless steel substrates (hereafter named Ti/SUS) and DLC monolithic films onto the stainless steel substrates (hereafter named DLC/SUS) were also respectively performed under the same sputtering conditions as for depositing the titanium layers and the DLC layers of the bi-layered films. Thus the deposition times for titanium films and DLC monolithic films was respectively of $5 \mathrm{~min}$ and of 22 min. The deposition by magnetron DC sputtering was performed under the condition that a titanium target was located at the distance of 90 $\mathrm{mm}$ just under a stainless steel substrate when a titanium layer was deposited onto the substrate, while a carbon target was also located at the distance of $90 \mathrm{~mm}$ just under a stainless steel substrate by shifting its position when a carbon layer was deposited onto the substrate. The schematic illustration of this magnetron DC sputtering system for the deposition of DLC/Ti/SUS is shown in Fig. 1.

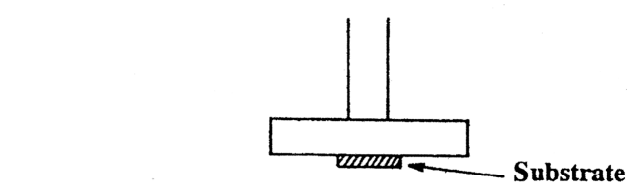

Ti target
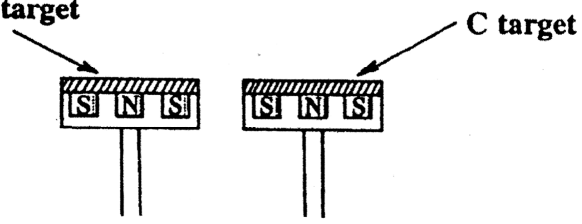

Fig.1. Schematic illustration of the magnetron DC sputtering system for the deposition of DLC/Ti bi-layered films onto stainless steel substrates.

The thickness of the deposited films was measured by tracing the substrate-film step using a surface roughness tester [7]. The surface morphology of the films was studied on SEM images.

The indentation hardness was measured using a dynamic-ultra-micro-hardness tester (SHIMADZU Corp. type DUH-201S) with a triangular pyramid-shaped indenter. Uploading tests following loading tests were performed to exclude the displacement brought about by the elastic deformation. Thus the dynamic hardness was calculated from the depth where the indenter was indented as a result of the plastic deformation. Furthermore the microstructures of the obtained DLC/Ti/SUS or DLC/SUS were characterized by Raman spectroscopy. The Raman spectra of the obtained films were measured using Renishaw inVia micro-Raman spectrometer with the $514.5 \mathrm{~nm}$ line of an Ar ion laser. The power of the laser was $10 \mathrm{~mW}$ and the laser was focused to about $1 \mu \mathrm{m}$ on the surface of the sample.

Potentiodynamic polarization experiments were carried out to evaluate the corrosion protection performance of obtained DLC/Ti/SUS with Hokuto Denko, HSV-100 system. The aqueous $0.05 \mathrm{M} \mathrm{NaCl}$ solution was used as an electrolyte, and a $\mathrm{Pt}$ sheet and a saturated calomel electrode (SCE) were used as counter electrode and a reference electrode, respectively. The scanning rate of potential was $10 \mathrm{mV} / \mathrm{sec}$.

\section{RESULTS AND DISCUSSION}

Both the obtained the DLC/Ti/SUS and the Ti/SUS appeared to be adhesive while the DLC/SUS peeled off partly. The thickness of the DLC/SUS, the Ti/SUS and the DLC/Ti/SUS were of $200 \mathrm{~nm}, 50 \mathrm{~nm}$ and $250 \mathrm{~nm}$, respectively. Figure 2 shows SEM images for the typical surface configuration of the DLC/Ti/SUS and of the DLC/SUS. Thus it was found that the DLC/Ti/SUS were more adhesive to the stainless steel than the DLC/SUS.

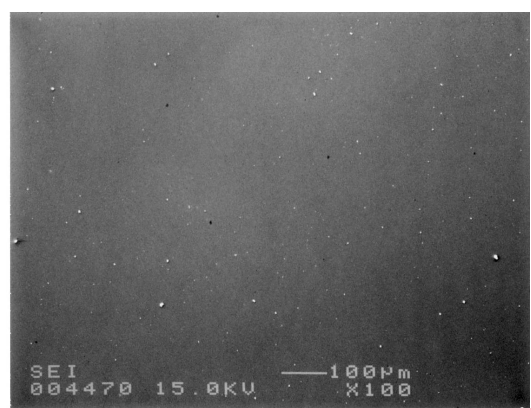

(a) DLC/Ti bi-layered film

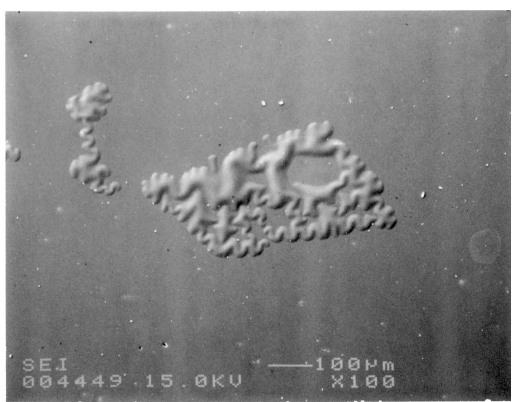

(b) DLC monolithic film

Fig.2. SEM micrographs for the typical surface configuration of the DLC/Ti bi-layered films (a) and of the DLC monolithic films (b) deposited onto stainless steel substrates. 
M.D.Bentzon et al. investigated the adhesion of various metallic inter-layers between steel and diamond-like carbon [8], and reported that bi-layers consisting of the combination of copper and a carbide-forming metal gave good adhesion since the copper might not only be able to bind effectively to the residual oxide layers on steel but also had good adhesion to carbide-forming metals. On the other hand, titanium is not only one of the carbide-forming metals but also its chemical reactivity is so high. Thus it was guessed that the titanium interlayer was rather adhesive to the DLC film and the good adhesion to the steel substrate was ascribed to such a high reactivity of titanium.

Raman spectrum of the obtained DLC/Ti/SUS is shown in Figure 3. A typical spectrum for DLC coatings was detected for the obtained DLC/Ti/SUS as well as for the DLC/SUS. Thus it was found that DLC coatings were also formed on the titanium layer.

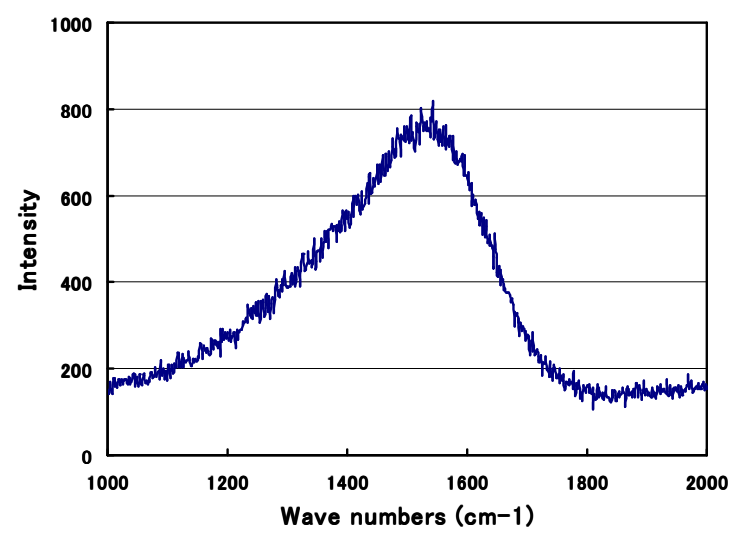

Fig.3. Raman spectrum of a DLC/Ti bi-layered film deposited by sputtering of a titanium target followed by sputtering a carbon one.

Figure 4 shows the dynamic hardness normalized by that of a stainless steel (SUS304), of a Ti/SUS, a DLC/Ti/SUS and a DLC/SUS, comparing with the dynamic hardness of the stainless steel substrate. The surface hardness of the DLC/Ti bi-layered films was much higher than that of the stainless steel substrates, while the surface hardness of the titanium films deposited on the stainless steel substrates was slightly lower than that of the stainless steel substrates. Thus it was found that the surface hardness of the stainless steel could be improved by coating it with the DLC/Ti bi-layered films.

The elastic modulus of titanium is twice lower than that of stainless steel (SUS304). Thus the titanium layer is effective in the relaxation of the internal stress in the DLC coatings such as the residual stress or the thermal stress. On the other hand, the thicker the DLC coating is, the higher the internal stress concentrated at the interface between the coating and the substrate is.
Moreover it is guessed that the surface hardness of DLC/Ti/SUS decreases with increasing the thickness of the titanium interlayer, because the hardness of titanium is much lower than that of the DLC coatings or the stainless steel, and because the titanium interlayer decreases the surface hardness of DLC/SUS, as shown above. Therefore the best conditions of the thickness of two layers of titanium and DLC for the improvement should be established through the optimal combination between the adhesion and the surface hardness specified by considering its application.

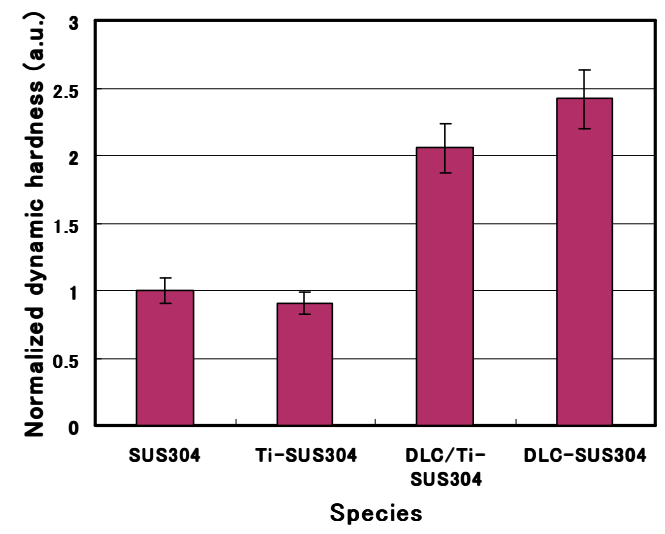

Fig.4. Dynamic hardness normalized by that of a stainless steel (SUS304), of a titanium film, a DLC/Ti bi-layered films and a DLC monolithic film deposited onto the stainless steel substrates.

The results of the potentiodynamic polarization experiments for the uncoated stainless steel (SUS304) substrate and the DLC/Ti/SUS are shown in Fig. 6.

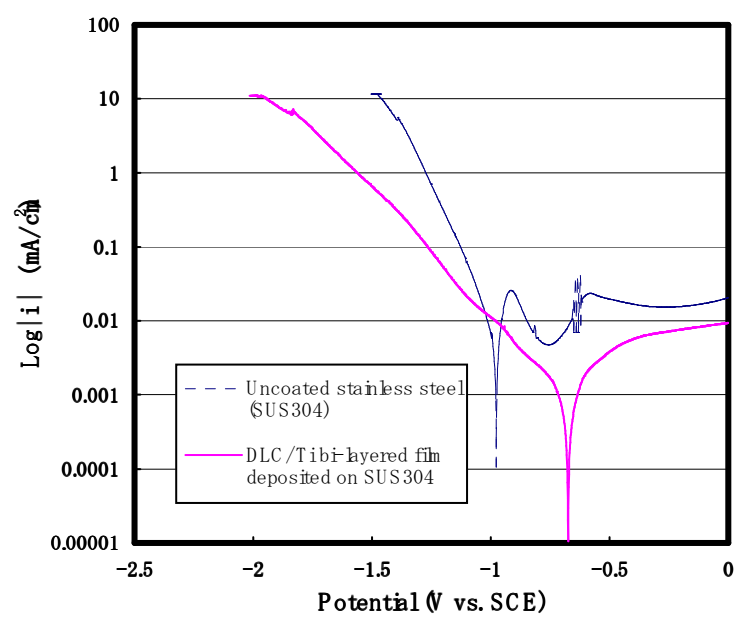

Fig.5. Potentiodynamic curves for the uncoated stainless steel (SUS304) substrate and the DLC/Ti bi-layered film deposited on the stainless steel substrate. 
The corrosion potential and the corrosion current of the uncoated stainless steel substrate were respectively estimated to be $-0.98 \mathrm{~V}$ and $0.005 \mathrm{~mA} / \mathrm{cm}^{2}$ from itspotentiodynamic curve by Tafel analysis [9]. On the other hand, the corrosion potential and the corrosion current of the DLC/Ti/SUS were respectively estimated to be $-0.68 \mathrm{~V}$ and $0.001 \mathrm{~mA} / \mathrm{cm}^{2}$ in the same manner. Thus it was found that the corrosion potential of the DLC/Ti/SUS was higher than that of the uncoated stainless steel by $0.3 \mathrm{~V}$ while the corrosion current of the DLC/Ti/SUS was 5 times smaller than that of the uncoated stainless steel. Therefore it was confirmed that the corrosion protective property of the stainless steel substrates could be improved by coating it with the DLC/Ti/SUS.

\section{CONCLUSIONS}

The deposition of DLC/Ti/SUS was carried out in the atmosphere of argon at the pressure of 0.4 $\mathrm{Pa}$ by sputtering of a titanium target followed by sputtering a carbon one. DLC layers and titanium layers were respectively deposited under the condition that the substrates were located right above the carbon target and the pure titanium target. DC powers supplied for the sputtering of the titanium target and the carbon one were respectively around $50 \mathrm{~W}$ and $300 \mathrm{~W}$. For the comparison, the sputter-deposition of DLC/SUS was also performed under the same sputtering conditions as for depositing the DLC layers of the bi-layered films.

Under visual observation, the obtained DLC/Ti/SUS appeared to be adhesive while the DLC/SUS deposited directly onto the substrates peeled off partly. Thus it was found that the DLC/Ti/SUS were more adhesive to the stainless steel than the DLC/Ti/SUS.

According to Raman spectroscopy, it was found that a typical spectrum for DLC coatings was detected for the obtained DLC/Ti/SUS, confirming that DLC coatings were also formed on the titanium layer.

According to indentation hardness measurements, the surface hardness of the DLC/Ti/SUS was much higher than that of the stainless steel substrates while the surface hardness of the Ti/SUS was slightly lower than that of the stainless steel substrates. Thus it was found that the surface hardness of the stainless steel could be improved by coating it with the DLC/Ti/SUS.

Furthermore, according to potentiodynamic polarization experiments, it was confirmed that the corrosion protective property of the stainless steel substrates could be also improved by coating it with the DLC/Ti/SUS.

\section{REFERENCES}

[1] K.L. Choy, E. Felix, Mater. Sci. Eng., A 278, 162-169(2000).

[2] A.A. Voevodin, J.S. Zabinski, Diamond Relat.
Mater. 7, 463-469 (1998).

[3] M. Allen, F. Law, N.Rushton, Clin. Mater. 17, 1-10(1994).

[4] S. Yang, D. Camino, A.H.S. Jones, D.G. Teer, Surf. Coat. Technol. 124, 110-118 (2000).

[5] O. Wanstrand, M. Larsson, P. Hedenqvist, Surf. Coat. Technol. 111, 247-252 (1999).

[6] M. Stuber, S. Ulrich, H. Leiste, A. Kratzsch, H. Holleck, Surf. Coat. Technol. 116, 591-598 (1999).

[7] M. Numoto, J. Surface Finishing Society of Japan, 40, 241-247(1989).

[8] M. D. Bentzon, K. Mogensen, J. Bindslev Hansen, C. Barholm-Hansen, C. Traeholt, P. Holiday, S. S. Eskildsen, Sur. Coat. Technol. 68(1994) 651.

[9] F. Stippich, E. Vera, G.K. Wolf, G. Berg, C. Friedrich, Surf. Coat. Technol. 103, 29-37(1998).

(Received January 31, 2011; Accepted March 30, 2011) 